\title{
Adapting to a new normal? 5 key operational principles for a radiology service facing the COVID-19 pandemic
}

\author{
Robert Chun Chen ${ }^{1} \cdot$ Thuan Tong Tan ${ }^{2} \cdot$ Lai Peng Chan ${ }^{1}$
}

Received: 11 March 2020 / Revised: 25 March 2020 / Accepted: 2 April 2020 / Published online: 23 April 2020

(C) European Society of Radiology 2020

\section{Key Points}

- Radiology services encountering the coronavirus disease-19 pandemic will need to modify their daily operational practices.

- Leadership, patient risk stratification, adequate manpower, operational workflow clarity, and workplace/social responsibility will help Radiology services safely and sustainably deal with the current disease outbreak.

Keywords COVID-19 $\cdot$ Coronavirus $\cdot$ Disease outbreak $\cdot$ Radiology $\cdot$ Singapore

Singapore's first case of coronavirus disease-19 (COVID-19) was confirmed on January 23, 2020, and treated at Singapore General Hospital [1]. Given its status as an international hub with close geographical and business ties to China, Singapore was a natural route of dissemination. Indeed, by early February 2020, our densely populated urban island nation had the second most number of confirmed COVID-19 cases outside of China [2]. At the onset, there was admittedly some panic, largely due to gaps in knowledge about the virus. Misinformation being circulated did not help; some early rumors suggested that the primary mode of spread was via aerosol transmission. Most evidence today suggests COVID-19 primarily spreads by way of large droplets [3].

Our radiology division is part of a large academic tertiary referral medical center, comprised of over 700 staff consisting of sub-specialty radiologists, trainees, radiographers, nurses, porters, and administrative staff. The relatively large organization required us to institute several initiatives, with an ultimate aim to ensure the safety of our patients and staff.

In any crisis, leadership and clear modes of communication are ingredients for success. We immediately set up a small radiology task force, consisting of key members of the

Robert Chun Chen

robert.chen@singhealth.com.sg

1 Department of Diagnostic Radiology, Singapore General Hospital, Outram Rd, Singapore 169608, Republic of Singapore

2 Department of Infectious Diseases, Singapore General Hospital, Singapore, Republic of Singapore division, inclusive of radiologists, radiographers, nurses, and administrative staff. The role of the radiology division task force was to review the daily operational capacity, inclusive of workload (Fig. 1a, b) and manpower, such that adjustments could be made quickly. Further, the task force would serve as a point of contact for those outside of the division, namely the larger umbrella hospital-wide disease outbreak task force (which radiology had direct representation with), as well as internally to the radiology division's ground staff. Several modes of direct communication between the task force and the ground staff were immediately created via instant messaging applications and email, allowing real-time clarifications and dissemination of information for speedy action.

The triage, categorization, and segregation of patients of varying risks during this pandemic continues to be an operational necessity (Fig. 2) [4]. Given the heterogeneous patient population coursing through any radiology department, definitions of how to categorize patients into non-suspect, suspect, and confirmed COVID-19 cases allow ground staff to understand which patients they can use standard precautions on versus those that require enhanced measures and additional personal protective equipment. In the earlier days of the outbreak, it was much easier to risk stratify patients; symptomatic patients with recent travel to Hubei Province or with occupational exposure to Chinese tourists were deemed at risk for COVID-19. With the recent global explosion of cases in Europe and America with known community spread, it is becoming more challenging to categorize patients into different risk categories and there may be a time in the near future when anybody coming into the radiology division may well be a possible COVID-19 case, and sustainable enhanced universal precautions are likely to become the "new normal." 
a

Total Weekly Radiology Workload

2019 vs 2020 (30 Dec - 21 Mar)

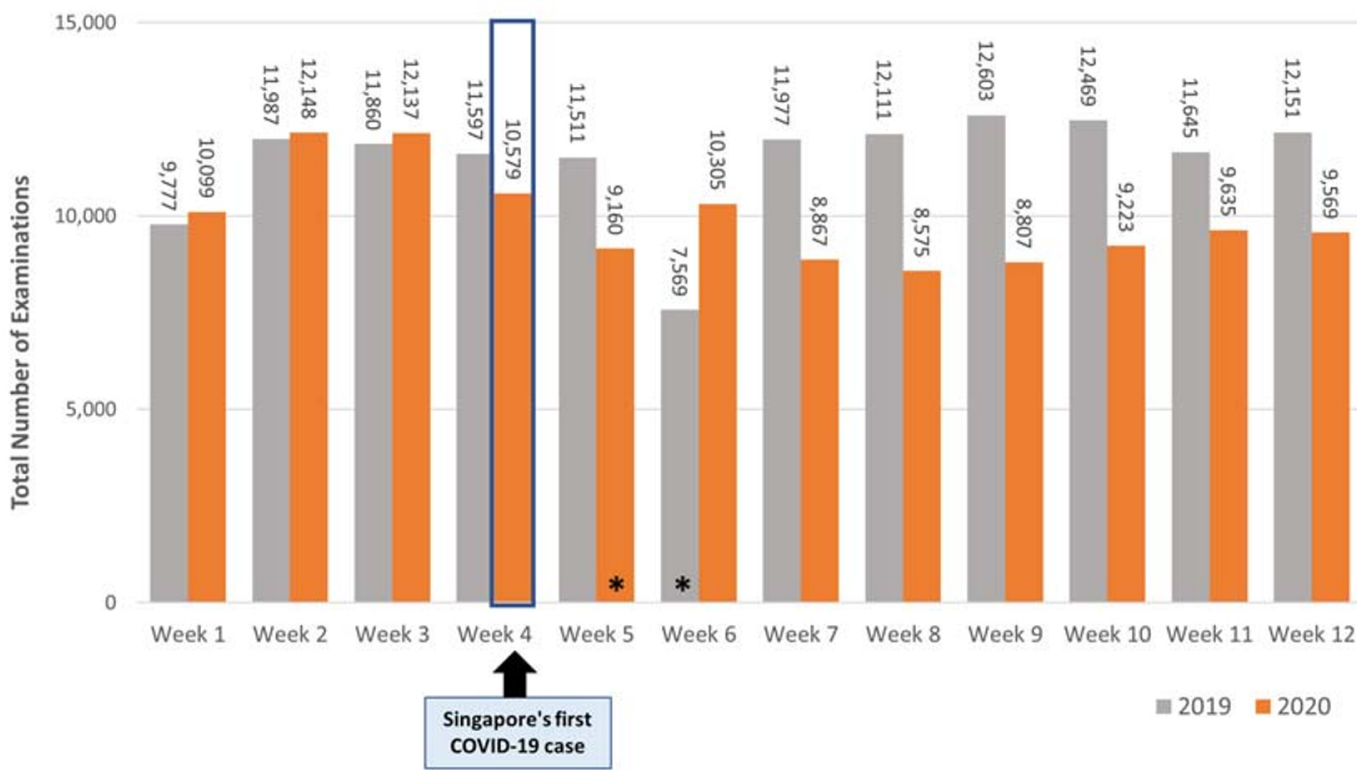

* Chinese New Year 2019 falls on Week 6 (5-6 Feb 2019) / Chinese New Year 2020 falls on Week 5 ( 25 -27 Jan 2020)

b

\section{COVID-19 Related Chest X-Ray Workload (20 Jan - 15 Mar 2020)}

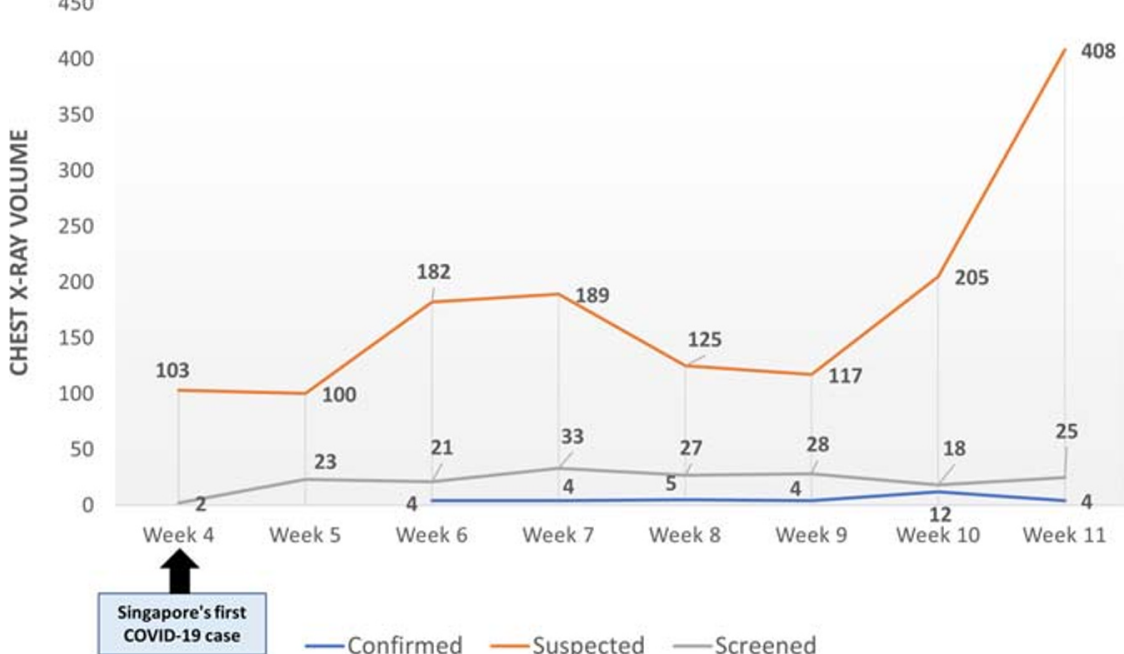

\section{Suspect Case Definition}

1. Clinical stigmata of flu-like symptoms AND within 14 days before onset of illness had travelled abroad; OR

2. Acute respiratory illness of any degree who, within 14 days before onset of illness had:

a. Been to areas requiring increased vigilance ${ }^{a} O R$

b. Been to any hospital abroad OR

c. Had close contact with a COVID-19 case $^{b}$ or is under national Quarantine Order

a Evolving list of countries based on global situation.

${ }^{b}$ Anyone who provided care e.g. healthcare worker

or lives with patient, or had close $(<2 \mathrm{~m})$ and

prolonged ( $>30 \mathrm{~min}$ ) contact e.g. shared a meal

Screen Case definition

Any one of the following:

1. Prolonged respiratory illness ( $>5$ days)

2. Referred by primary care physician

3. Stays in congregate setting (e.g. Dormitory, nursing home)

4. Visited confirmed local clusters

5. High-risk occupations (e.g. Healthcare worker,

immigration staff)
Fig. 1 a Total radiology workload, 2019 vs 2020. We have made a conscious effort to reduce our workload to deal with the increased operational demands and potential surges in patient load during this outbreak period. Since our first COVID-19 case, our workload has decreased by approximately $25 \%$ compared to last year. b COVID-19-

Ensuring adequate manpower to deal with the crisis must be addressed. We ultimately made the difficult decision of indefinitely postponing selected staffs' leave to ensure there was adequate manpower on the ground. Further, we segregated into 2 to 3 functional teams, so in the case of a quarantine situation disabling 1 functional subunit, the other team related chest x-ray workload, 20 January to 15 March 2020. Our COVID-19 suspect chest x-ray workload has dramatically increased, largely related to an interim change in suspect case definition from recent travel from China to any recent travel from abroad

could independently carry on the needs of the radiology division [5]. Splitting into smaller subunits admittedly has taken a toll on some of staff. Even prior to the COVID-19 outbreak, we were generally short staffed of radiographers, and their longer 12-h shifts during this outbreak has been a challenge. 
Radiology Entrance Triage Questionnaire

Declaration by Patient / Visitor

Please circle the appropriate answers

\begin{tabular}{|l|l|c|}
\hline 1. & $\begin{array}{l}\text { Have you travelled abroad (i.e. to any countries outside of Singapore) } \\
\text { in the past 14 days? }\end{array}$ & Yes / No \\
\hline 2. & Do you have flu-like symptoms (e.g. fever, cough etc.)? & Yes / No \\
\hline 3. & $\begin{array}{l}\text { Did you, in the past 14 days, come in close contact with someone } \\
\text { who: } \\
\text { (i) Is a confirmed COVID-19 case; OR } \\
\text { (ii) Is part of a coVID-19 cluster? }\end{array}$ & Yes / No \\
\hline
\end{tabular}

\begin{tabular}{|l|l|}
\hline \multicolumn{1}{|c|}{ Action } & \multicolumn{1}{|c|}{ Give Mask? } \\
\hline $\begin{array}{l}\text { 1) Give yellow sticker } \\
\text { and allow patient to } \\
\text { enter; OR }\end{array}$ & 1) Yes; OR \\
2) No \\
$\begin{array}{l}\text { Physician to conduct } \\
\text { secondary screening }\end{array}$ & \\
\hline
\end{tabular}

I hereby declare that the above statements are true, accurate and complete*.

*Anyone making a false declaration can be prosecuted under Section 21(A) of the Infectious Diseases Act.

Patient's / Visitor's Signature

Fig. 2 Radiology entrance triage questionnaire. If a patient does not have any flu-like symptoms, they are allowed to enter our radiology department and continue with their imaging examination. If a patient has flulike symptoms, they are immediately brought to a separate room. A physician will take into account any recent travel history from high risk COVID-19 countries, contact with known local COVID-19 case/clusters,

How have our daily radiology operations been affected? For non-suspect COVID-19 patients, we continue to use our pre-pandemic standard precautions, ensuring surface cleaning of patient contact points with standard anti-microbial wipes, such as the x-ray buckys, ultrasound probes, and CT couches after each patient. Strict hand hygiene practices are already part of our normal pre-pandemic routine; our only additional measure is that all our patient-fronting staff wear surgical masks. Our daily throughput for this group of patients have not been affected.

For suspect or confirmed COVID-19 patients, our basic tenet has been to minimize unnecessary imaging, reducing the potential spread of pathogen to other patients and staff. However, PCR testing may not be sensitive enough to exclude COVID-19, and many patients may need radiological investigations prior to disease confirmation $[6,7]$. To image these patients, we have taken additional safety measures at the expense of patient throughput. For general radiography and ultrasound, we have leveraged upon the use of portable examinations to minimize patient movement. For examinations, we lack portable capability for, including MRI and CT, carefully planned routes of transportation ensure minimal spread of pathogen and contact with personnel [4]. Simulation of these transfers have helped identify gaps in the process and should be carefully rehearsed. Further, as far as possible, we have performed examinations for all suspect or confirmed cases in negative pressure CT and MRI rooms, installed in response to our previous 2003 severe acute respiratory syndrome (SARS) pandemic, and use full personal protective equipment [8]. Meticulous vetting of examinations is being made to ensure examinations are being done appropriately and add value to and exam urgency before deciding whether or not to allow them to proceed with their radiology examination. Note every patient must sign on the declaration form, and providing false statements is subject to a fine of up to SGD $\$ 10,000$ or jail up to 6 months or both (in accordance with Section 21(A) of the Singaporean Infectious Diseases Act)

patient care [4]. Decontamination for portable x-ray machines includes surface cleaning to the entire machine with standard anti-microbial wipes. Our CT procedure for COVID-19 patients includes wrapping potential contact areas (gantry, couch, injector controls) with plastic covering; after the plastic is removed, we use standard anti-microbial wipes to clean the machine, and then proceed with terminal cleaning and UV-C irradiation to the scan room. The decontamination process for portable $\mathrm{x}$-ray and CT takes $20 \mathrm{~min}$ and $90 \mathrm{~min}$, respectively.

Workplace and social responsibility continues to be advocated. Posters and videos demonstrating proper hand hygiene technique and donning and doffing of personal protective equipment are clearly visible in staff areas in our division and posted on our intranet [5]. If people are sick, they are advised to stay home and avoid social activities. Since we have segregated into smaller functional teams at work, minimal mixing with counterpart teams at break time and after work is essential for our manpower segregation concept to be of any utility. Safe distancing, defined by standing at least $1 \mathrm{~m}$ apart, both in and outside of the workplace is known to decrease the possibility of pathogen spread [9]. In line with published recommendations, we have instituted mandatory temperature reporting via a secure weblink of all our staff twice a day, 7 days a week, using a hospital issued thermometer [10].

As the COVID-19 situation continues to evolve, we will continue to persevere and hope that the concepts we have introduced will allow us to contain and nimbly adapt to the situation as it unfolds. It is possible that this pandemic may be a new normative state, and we may have to develop new infection control standards such that operations can continue 
with minimal risk to staff and patients. At the time of writing (26 March 2020), we have had 35 confirmed COVID-19 cases in our hospital, 9 of which have safely discharged, 1 transferred, and 25 remaining in the isolation unit; no intra-hospital transmission to health care workers has occurred. Our experience over the past 9 weeks may help to shape the response of other radiology services during this rapidly evolving global COVID-19 pandemic.

Acknowledgments We thank Dr. Tan Bien Soo, Dr. Lionel Cheng, Dr. Lim Kheng Choon, Dr. Nanda Kumar Karaddi Venkatanarasimha, Li Sheng, Sim Wei Yow, Ooi Chin Chin, Hong Wei Liang, Pei Yuan Chang, Tiong Mee Ling, Thong Mei Chuen, and all the members of the Division of Radiological Sciences and all our colleagues at the Singapore General Hospital for their heart and commitment during this COVID-19 pandemic.

Funding information The authors state that this work has not received any funding.

\section{Compliance with ethical standards}

Guarantor The scientific guarantor of this publication is Robert Chun Chen.

Conflict of interest The authors of this manuscript declare no relationships with any companies whose products or services may be related to the subject matter of the article.

Statistics and biometry No complex statistical methods were necessary for this paper.

Informed consent Written informed consent was not required for this study because it is an operational editorial.

Ethical approval Institutional Review Board approval was not required because it is an operational editorial.

Study subjects or cohorts overlap Not relevant.

Methodology

- Not relevant

\section{References}

1. Ministry of Health Singapore (2020) Coronavirus disease (COVID19). Available via https://www.moh.gov.sg/news-highlights/details/ confirmed-imported-case-of-novel-coronavirus-infection-insingapore-multi-ministry-taskforce-ramps-up-precautionarymeasures. Accessed 9 Mar 2020

2. Straits Times Singapore (2020) Coronavirus. Available via https:// www.straitstimes.com/singapore/health/coronavirus-4-moreconfirmed-cases-in-singapore-28-cases-so-far. Accessed 9 Mar 2020

3. Centers for Disease control and Prevention (2020) Coronavirus disease (COVID-19). Available via https://www.cdc.gov/ coronavirus/2019-ncov/about/transmission.html. Accessed 26 Mar 2020

4. Da Zhuang K, Tan BS, Tan BH, Too CW, Tay KH (2020) Old threat, new enemy: is your interventional radiology service ready for the coronavirus disease 2019? Cardiovasc Intervent Radiol. https://doi.org/10.1007/s00270-020-02440-6

5. Cheng LTE, Chan LP, Tan BH et al (2020) Déjà vu or Jamais vu? How the severe acute respiratory syndrome experience influenced a Singapore Radiology Department's response to the coronavirus disease (COVID-19) pandemic. AJR Am J Roentgenol. https://doi. org/10.2214/AJR.20.22927

6. Ai T, Yang ZL, Hou HY et al (2020) Correlation of chest CT and RT-PCR testing in coronavirus disease 2019 (COVID-19) in China: a report of 1014 cases. Radiology. https://doi.org/10.1148/radiol. 2020200642

7. Pan Y, Guan H, Zhou S et al (2020) Initial CT findings and temporal changes in patients with the novel coronavirus pneumonia (2019nCov): a study of 63 patients in Wuhan, China. Eur Radiol. https:// doi.org/10.1007/s00330-020-06731-x

8. Gogna A, Tay KH, Tan BS (2014) Severe acute respiratory syndrome: 11 years later-a radiology perspective. AJR Am J Roentgenol 203:746-748

9. Markel H, Lipman HB, Navarro JA et al (2007) Nonpharmaceutical interventions implemented by US cities during the 1918-1919 influenza pandemic. JAMA 6:644-654

10. Huang C, Wang Y, Li X et al (2020) Clinical features of patients infected with 2019 novel coronavirus in Wuhan, China. Lancet. https://doi.org/10.1016/S0140-6736(20)30183-5

Publisher's note Springer Nature remains neutral with regard to jurisdictional claims in published maps and institutional affiliations. 